\title{
Diversity and structure of the tree community of a fragment of tropical secondary forest of the Brazilian Atlantic Forest domain 15 and 40 years after logging
}

\author{
ARY T. OLIVEIRA FILHO',4, DOUGLAS A. CARVALHO ${ }^{2}$, ENIVANIS A. VILELA², \\ NILTON CURI ${ }^{3}$ and MARCO AURÉLIO L. FONTES ${ }^{1}$
}

(received: August 28, 2003; accepted: July 22, 2004)

\begin{abstract}
Diversity and structure of the tree community of a fragment of tropical secondary forest of the Brazilian Atlantic Forest domain 15 and 40 years after logging). Two adjacent tracts of tropical secondary forest, situated in Itambé do Mato Dentro, south-eastern Brazil, which had been regenerating for 15 and 40 years after clearing, were compared with the purpose of detecting differences in species diversity and composition, species guild composition (regeneration, stratification and dispersion), and stand structure. Four and three 1,125 $\mathrm{m}^{2}$ plots laid on the 15- and 40-year-old stands, respectively, sampled 2,430 trees with diameter at the base of the stem $\geq 5 \mathrm{~cm}$. The number of species $(\mathrm{S}=199)$ was high for this forest type and significantly higher for the older stand. Tree density was significantly higher in the younger stand, particularly for smaller trees, whereas the two stands did not differ in both basal area and volume per hectare. Trees of shade-tolerant and understory species were significantly more abundant in the older stand. Though sharing a large proportion of species (49\%), the two stands differed significantly in the abundance of many species. Live stumps probably contributed to the relatively quick restoration of some forest characteristics, particularly species diversity, basal area and volume.
\end{abstract}

Key words - Brazilian Atlantic forest, forest regeneration, succession, tropical secondary forest

RESUMO - (Diversidade e estrutura da comunidade arbórea de um fragmento de floresta tropical secundária do domínio da Floresta Atlântica 15 e 40 anos após o corte). Dois trechos adjacentes de floresta secundária, situados em Itambé do Mato Dentro, MG, e que estiveram em regeneração por 15 e 40 anos depois do corte foram comparados com o propósito de detectar diferenças em diversidade e composição de espécies, composição de guildas (regeneração, estratificação e dispersão) e estrutura do povoamento. Quatro e três parcelas de $1.125 \mathrm{~m}^{2}$ foram distribuídas nos povoamentos de 15 e 40 anos de idade, respectivamente, e amostraram 2.430 árvores com diâmetro na base do tronco $\geq 5 \mathrm{~cm}$. O número de espécies $(S=199)$ foi alto para essa tipologia florestal e significativamente maior para o povoamento mais velho. A densidade de árvores foi significativamente mais alta no povoamento mais jovem, particularmente para árvores menores, ao passo que os dois povoamentos não diferiram em área basal e volume por hectare. Árvores de espécies tolerantes à sombra e de espécies de subbosque foram significativamente mais abundantes no povoamento mais velho. Apesar de compartilharem uma grande proporção de espécies (49\%) os dois povoamentos diferiram significativamente quanto à abundância de muitas espécies. As cepas das árvores provavelmente contribuíram para a relativamente rápida restauração de algumas características da floresta, particularmente diversidade de espécies, área basal e volume.

Palavras-chave - Floresta Atlântica, floresta secundária tropical, regeneração florestal, sucessão

\section{Introduction}

The Brazilian Atlantic forests, which once covered an area of about 1.1 million $\mathrm{km}^{2}$ along the eastern Brazilian coast, are among the most threatened tropical forests of the world, as their range largely coincides with the most populated areas of the country (SOS Mata

1. Universidade Federal de Lavras, Departamento de Ciências Florestais, 37200-000 Lavras, MG, Brasil.

2. Universidade Federal de Lavras, Departamento de Biologia, 37200-000 Lavras, MG, Brasil.

3. Universidade Federal de Lavras, Departamento de Ciência do Solo, 37200-000 Lavras, MG, Brasil.

4. Corresponding author: ary@ufla.br
Atlântica \& INPE 1993). These forests are now reduced to only about $5 \%$ of their original cover and most remnants are in either small $(<10$ ha) disturbed fragments or larger areas sheltered on steep mountain slopes (Viana \& Tabanez 1996, SOS Mata Atlântica 1998).

During the last decades, the increasing pressure on authorities to halt indiscriminate deforestation has achieved results and fragments of secondary forests are thriving all over the region. However, the 'complete' restoration of many characteristics of the original mature forest is dependent upon many factors, particularly regeneration time, distribution and size of forest fragments, the history of land use and disturbance, and the existence of preserved mature forests on the 
landscape (Whitmore 1990, Saldarriaga \& Uhl 1991, Guariguata \& Dupuy 1997, Parthasarathy 1999). Important forest characteristics, such as species richness and diversity, species composition, and tree density and biomass, are affected differently by these factors and are restored at different rates (Uhl et al. 1982, Saldarriaga et al. 1988, Saldarriaga \& Uhl 1991, Tabarelli \& Mantovani 1999).

A detailed knowledge of the mechanisms of tree community organization that take place during forest regeneration is necessary to provide a solid foundation for an efficient management and conservation of tropical secondary forests remnants. There are, however, few studies of forest regeneration in the Brazilian Atlantic Forest domain and most that have been done are in the coastal rainforests (e.g. Klein 1980, Mendonça et al. 1992, Pessoa et al. 1997, Tabarelli \& Mantovani 1999). In the present contribution we compared a number of characteristics of two adjacent tracts of hinterland semideciduous forest of the Atlantic Forest Domain. By the time of the study, these forest stands had been regenerating for 15 and 40 years after clearing, respectively. We posed the following central question: were there clear differences between the two stands in their tree community features, and, if so, could these differences be related to regeneration time? To this goal, we compared the two stands for their species richness and evenness, species composition, proportion of trees per species guild (regeneration, stratification and dispersion), and stand structure (tree density, basal area, volume and size class distribution).

\section{Material and methods}

Study area - Our study was carried out in a ca. 9.8 ha forest fragment located at $19^{\circ} 26^{\prime} \mathrm{S}$ and $43^{\circ} 14^{\prime} \mathrm{W}$, and at $610-630 \mathrm{~m}$ of altitude, in Fazenda Camarinha, municipality of Itambé do Mato Dentro, state of Minas Gerais, Brazil. The area lies on the margin of the Dona Rita Hydroelectric Reservoir, formed by a dam built across the Rio Tanque in 1960 . The climate of the region is characterised by warm temperatures throughout the year with a rainy summer and a dry winter. The tree flora of this area is characteristic of the semideciduous forests of the Rio Doce basin, in eastern Minas Gerais (Carvalho et al. 2000, Oliveira-Filho \& Fontes 2000). Small forest fragments at different regeneration phases are abundant in the region and the five nearest fragments are found within a $1 \mathrm{~km}$ distance from the studied forest.

Different areas of the forest fragment were logged on three occasions, as indicated in Figure 1. The first harvest was in 1957 during the construction of the dam. All trees on the valley bottom (now under water) and on part of the surrounding slopes (western side) were felled and used for props by the builders. After the reservoir was formed, the forest regenerated on the felled slope and there was little disturbance by humans in the fragment for more than 20 years. After this period, the fragment was harvested twice more and the remaining areas of mature forest were logged to produce charcoal. The first logging, in 1982, cleared the eastern side of the slope and the second, in 1996, the hilltop. Therefore,

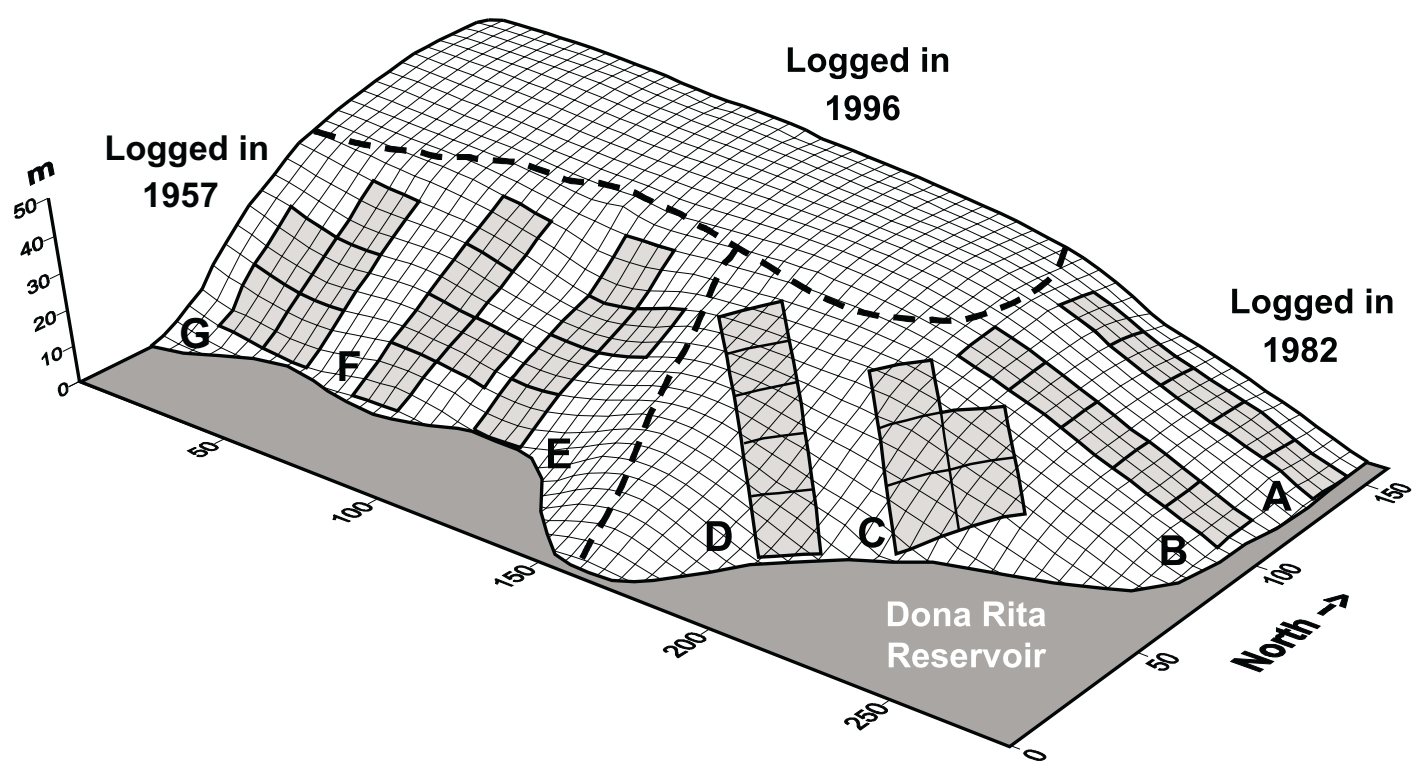

Figure 1. Surface grid of the area of secondary forest surveyed in Itambe do Mato Dentro, south-eastern Brazil, showing the distribution of the seven sample plots (A-G) and the three sectors (dashed lines) where trees were felled in different years. Plots are made up of five adjacent $15 \times 15 \mathrm{~m}$ subplots; lines on surface grid are spaced at $5 \mathrm{~m}$ intervals. 
when we surveyed the forest in 1997, there were three sectors corresponding to different regeneration ages. The stands on the western (ca. 2.1 ha) and eastern (ca. 3.2 ha) slopes were 40 and 15 years old, respectively, and forest physiognomy was characterised in both stands by a canopy of irregular height (12-20 m), dense understory and low deciduousness. During the dry season $<15 \%$ of trees are totally leafless while the others only reduce their foliage. The hilltop vegetation (ca. 4.5 ha) was only one-year old and consisted of a dense mix of saplings, sprouting stumps and vine tangles. Forest and soil surveys - Sampling procedure followed the protocol of our previous studies (e.g. Botrel et al. 2002) devised to capture the maximum variation in both species abundance distributions and environmental variables. We sampled soils and trees in seven $1,125 \mathrm{~m}^{2}$ plots located in the forest fragment, four plots in the 15-year-old sector and three in the 40-year-old (Figure 1), in order to use a similar sampling intensity for both stands (ca. 15\% of their areas). The hilltop sector was not included because logging was too recent and there was no tree above the minimum sampling size established. The area was also divided for sampling into three slopes, according to their aspect: east (plots A and B), southeast (plots C and D) and south (plots E, F and G). On each slope, plots were aligned perpendicularly to the reservoir margin and $20 \mathrm{~m}$ apart. Each plot was made up of five $15 \times 15$ $m$ adjacent subplots arranged in a row starting at the margin of the reservoir and ending at the forest edge adjacent to the hilltop. In four of the plot areas this distance was $<75 \mathrm{~m}$, therefore some subplots were placed to the side of the main rows, at a randomly assigned position along the row.

All trees occurring in the subplots with a circumference at the base of the stem (CBS) $\geq 15.7 \mathrm{~cm}$ ( $5 \mathrm{~cm}$ diameter) were identified and their CBS and total height were measured. For the few buttressed trees, the CBS was recorded above the buttresses. Voucher specimens are stored at the Herbarium of the Universidade Federal de Lavras (ESAL). The species of flowering plants (all but Cyatheaceae) were classified into families following the Angiosperm Phylogeny Group II (APG II 2003).

In order to assess differences in soil properties between the two stands, four 0.5-litre soil samples were collected from a depth of $0-20 \mathrm{~cm}$ at the midpoints between the corners and the centre of each subplot. The four soil samples were mixed up to produce a single soil sample representative of the subplot surface soil. Chemical and granulometric analyses were carried out at the Soil Laboratory of the Universidade Federal de Lavras following standard procedures (Embrapa 1997).

Data analysis - Plot means were calculated for each of the following soil variables: $\mathrm{pH}, \mathrm{P}, \mathrm{K}^{+}, \mathrm{Ca}^{++}, \mathrm{Mg}^{++}, \mathrm{Al}^{+++}$, total exchangeable bases, saturation of bases, organic matter, and percentage sand, silt and clay. Above ground tree volume estimates were calculated for each plot using the formula 'volume = basal area*height*0.6', following Korning \& Balslev (1994). Tree frequency distributions into classes of diameter and height were prepared for each separated sample plot using class intervals with exponentially increasing ranges to make up for the normally steep decrease in tree density toward larger diameters and heights (see Oliveira Filho et al. 2001). Then each soil variable and tree community physiognomic variable was compared between the two stands, in a $3 \times 4$ plots design, using two-sample $t$ tests. Independent, normal populations with equal variances were deliberately assumed to perform the tests. As stated by Zar (1996), "the biological researcher cannot (...) always be assured that these assumptions are correct. Fortunately numerous studies have shown that the $t$ test is robust enough to stand considerable departures from its theoretical assumptions, especially if sample sizes are equal or nearly equal, and especially when two-tailed hypotheses are considered".

Three parameters of species diversity were used to compare the 15- and 40-year-old stands: species richness, Shannon diversity index $\left(H^{\prime}\right)$ and Pielou evenness $\left(J^{\prime}\right)$ (Krebs 1989). These parameters were plotted on sample area for each of the two stands using the $15 \times 15 \mathrm{~m}$ subplots as sample units in order to refine the effects of increasing area. The means and their $95 \%$ confidence intervals of each diversity parameter were calculated for each sample size from subsampling all possible combinations of subplots. The secondorder jackknife estimator (Palmer 1991) of the total number of species in each stand was also calculated. The Shannon diversity indices of the two stands were compared with Hutcheson's $t$ test (Zar 1996).

A detrended correspondence analysis, DCA (Hill \& Gauch 1980), was performed using the program Canoco 4 (ter Braak \& Šmilauer 1998) in order to investigate whether the emerging patterns of species abundance distribution were related to the two stand ages. The 35 subplots were used, instead of the seven plots, in order to assess both intra-plot and inter-plot variations. The species abundance matrix consisted of the relative importance values (RIVs) of each species across subplot. RIVs were obtained from the sum of species density and volume, both standardised to the site total (van Tongeren 1995). As recommended by ter Braak (1995), rare species were eliminated (including only the 47 species with RIV $\geq 5$ in the total sample) and RIVs were log-transformed before analysis. The seven stands were graphically discriminated on the subplot ordination diagram in order to assess, a posteriori, the relationship between the overall species distribution pattern and the two stand ages.

For each of the 47 species used in DCA, their abundances expressed as both number of trees and total volume were compared between the 15- and 40-year-old stands. Chi-square statistics was used to test the species frequency of trees in each stand against the expected frequencies obtained from the total number of trees of each stand, and the $t$ test to compare the distribution of the species volume in each stand in a $4 \times 3$ plots design (see assumptions of the $t$ test above). 
In order to search for ecologically meaningful differences between the two forest communities for particular species groups, all 201 species were classified into three guild systems: regeneration, stratification and dispersion. The regeneration guilds, based on Swaine \& Whitmore's (1988) ecological species groups, were: (a) pioneer, (b) shadetolerant climax species, and (c) light-demanding climax species. Pioneer and climax species differ on the light environment required for establishment, the former depending on direct sunlight. Light-demanding and shade-tolerant climax species are actually the halves of a continuum of solar radiation required by the plants for release from the bank of juveniles. The stratification guilds were based on the stature commonly reached by the adult individuals: (a) small, 2-8 m; (b) medium, $>$ 8-17.5 m; and (c) large, $\geq 17.5 \mathrm{~m}$ tall (see Oliveira-Filho et al. 1994). The dispersion guilds were: (a) anemochorous, species with mechanisms to facilitate dispersal by wind; (b) zoochorous, species with characteristics related to dispersal by animals; and (c) autochorous, species dispersed by free fall or ballistic mechanisms (van der Pijl 1982). The classification of each species into the species guilds was based on scientific knowledge registered in the literature (Morellato \& Leitão-Filho 1992, Lorenzi 1992, 1998, Gandolfi et al. 1995, Oliveira-Filho et al. 1997, Barroso et al. 1999, Nunes et al. 2003). The distribution of trees into the species guilds in the two forest communities were tested for independence with chi-square tests for contingency tables (Zar 1996).

\section{Results}

The soils of the two stands did not differ significantly for all chemical and textural variables but organic matter (table 1). The proportions of organic matter were significantly higher in the soils of the 15 -year-old forest stand than in those of the 40-year old stand.

Tree density was significantly higher in the younger stand than in older one; no significant difference was found between the stands for basal area and volume per hectare (table 2). The distribution of the number of trees per plot differed significantly between the two stands for particular classes of diameters and heights (figure 2). The younger stand had significantly higher numbers of trees of the $5-9 \mathrm{~cm}$ diameter class and > 5-10 m height class.

A total of 2.430 individual trees and 199 species were registered (table 3 ). The numbers of species sampled for each stand age were similar, 152 and 145 for the younger and older stands, respectively (table 2), and 98 species (i.e. 49\%) were shared by the two stands. Despite these figures, the shapes of the two speciesarea curves (figure $3 \mathrm{~A}$ ) indicate that the 15-year old subplots accumulated significantly more species at smaller areas ( $\leq 7$ subplots) but were surpassed by the 40-year old subplots over larger sample areas $(\geq 12$ subplots), suggesting that total species richness was probably higher in the older stand. In fact, at the sample size of 14 subplots, the $95 \%$ confidence intervals obtained from all possible subplot series were 135.9-136.1 and 140.2-142.0 species for the younger and older stands, respectively. This was reinforced by the second-order jackknife estimators, which projected a total of 227.2 species for the older stand in contrast to 215.8 species for the younger stand (table 2).

The evenness-area curves in figure $3 \mathrm{~B}$ show a steeper decrease of $J$ ' values with increasing sample size for the younger stand. However, this difference

Table 1. Soil variables in the two stands of secondary forest in Itambé do Mato Dentro, south-eastern Brazil. Figures are means \pm standard deviations of the four and three $1,125 \mathrm{~m}^{2}$ plots used to survey the 15 - and 40-year-old stands, respectively. Each variable is compared between plots using two-sample $t$ tests. (ns = non-significant).

\begin{tabular}{|c|c|c|c|}
\hline & $\begin{array}{c}15 \text {-year-old stand } \\
N=4\end{array}$ & $\begin{array}{c}\text { 40-year-old stand } \\
\quad N=3\end{array}$ & $t$ tests \\
\hline $\mathrm{pH}$ in $\mathrm{H}_{2} \mathrm{O}$ & $4.44 \pm 0.02$ & $4.37 \pm 0.19$ & $0.62 \mathrm{~ns}$ \\
\hline P Mehlich $\left(\mathrm{mg} . \mathrm{dm}^{-3}\right)$ & $1.4 \pm 0.00$ & $1.5 \pm 0.1$ & $2.00 \mathrm{n} \mathrm{s}$ \\
\hline $\mathrm{K}+\left(\mathrm{mg} \cdot \mathrm{dm}^{-3}\right)$ & $46.0 \pm 5.2$ & $41.5 \pm 10.8$ & $0.62 \mathrm{~ns}$ \\
\hline $\mathrm{Ca}++\left(\right.$ cmolc $\left.\cdot \mathrm{dm}^{-3}\right)$ & $0.36 \pm 0.04$ & $0.57 \pm 0.27$ & $1.37 \mathrm{n} \mathrm{s}$ \\
\hline $\mathrm{Mg}++\left(\mathrm{cmolc} \cdot \mathrm{dm}^{-3}\right)$ & $0.12 \pm 0.03$ & $0.28 \pm 0.23$ & $1.21 \mathrm{n} \mathrm{s}$ \\
\hline $\mathrm{Al}+++\left(\mathrm{cmolc} \cdot \mathrm{dm}^{-3}\right)$ & $1.94 \pm 0.08$ & $1.71 \pm 0.38$ & $1.00 \mathrm{n} \mathrm{s}$ \\
\hline Total bases $\left(\mathrm{cmolc} \cdot \mathrm{dm}^{-3}\right)$ & $0.60 \pm 0.07$ & $0.96 \pm 0.50$ & $1.24 \mathrm{n} \mathrm{s}$ \\
\hline Saturation of bases (\%) & $5.68 \pm 0.88$ & $11.00 \pm 6.74$ & $1.35 \mathrm{~ns}$ \\
\hline Organic matter (\%) & $3.06 \pm 0.14$ & $2.32 \pm 0.06$ & $7.17 p<0.01$ \\
\hline Sand $(\%)$ & $50.2 \pm 1.2$ & $50.9 \pm 5.4$ & $0.20 \mathrm{~ns}$ \\
\hline Silt (\%) & $18.3 \pm 2.7$ & $17.8 \pm 1.2$ & $0.25 \mathrm{~ns}$ \\
\hline Clay $(\%)$ & $32.2 \pm 3.1$ & $31.3 \pm 5.5$ & $0.23 \mathrm{~ns}$ \\
\hline
\end{tabular}


Table 2. Variables of tree community physiognomy and diversity in the two stands of secondary forest in Itambé do Mato Dentro, south-eastern Brazil. Figures with ranges are means \pm standard deviations of the four and three $1,125 \mathrm{~m}^{2}$ plots used to survey the 15- and 40-year-old stands, respectively. Each variable is compared between plots using two-sample $t$ tests (Hutcheson's $t$ is used for Shannon's diversity, $H^{\prime}$ ).

\begin{tabular}{lcccc}
\hline & $\begin{array}{c}\text { Total Sample } \\
N=7\end{array}$ & $\begin{array}{c}\text { 15-year-old stand } \\
N=4\end{array}$ & $\begin{array}{c}\text { 40-year-old stand } \\
N=3\end{array}$ & $t$ statistics \\
\hline Number of trees per plot & $347 \pm 78$ & $401 \pm 42$ & $276 \pm 49$ & $3.07 *$ \\
Basal area per plot $\left(\mathrm{m}^{2}\right)$ & $4.256 \pm 0.182$ & $4.318 \pm 0.088$ & $4.174 \pm 0.264$ & $0.87 \mathrm{~ns}$ \\
Volume per plot $\left(\mathrm{m}^{3}\right)$ & $32.02 \pm 3.76$ & $30.03 \pm 2.31$ & $34.67 \pm 3.98$ & $1.65 \mathrm{~ns}$ \\
Total number of trees & 2430 & 1603 & 827 & \\
Total number of species & 199 & 152 & 145 & $0.31 \mathrm{~ns}$ \\
Shannon's $H^{\prime}$ (nats.individual $\left.{ }^{-1}\right)$ & 4.317 & 4.123 & 4.105 & \\
Pielou's evenness, $J^{\prime}$ & 0.816 & 0.821 & 0.825 & \\
Second-order jackknife estimate & 268 & 216 & 227 & \\
\hline
\end{tabular}
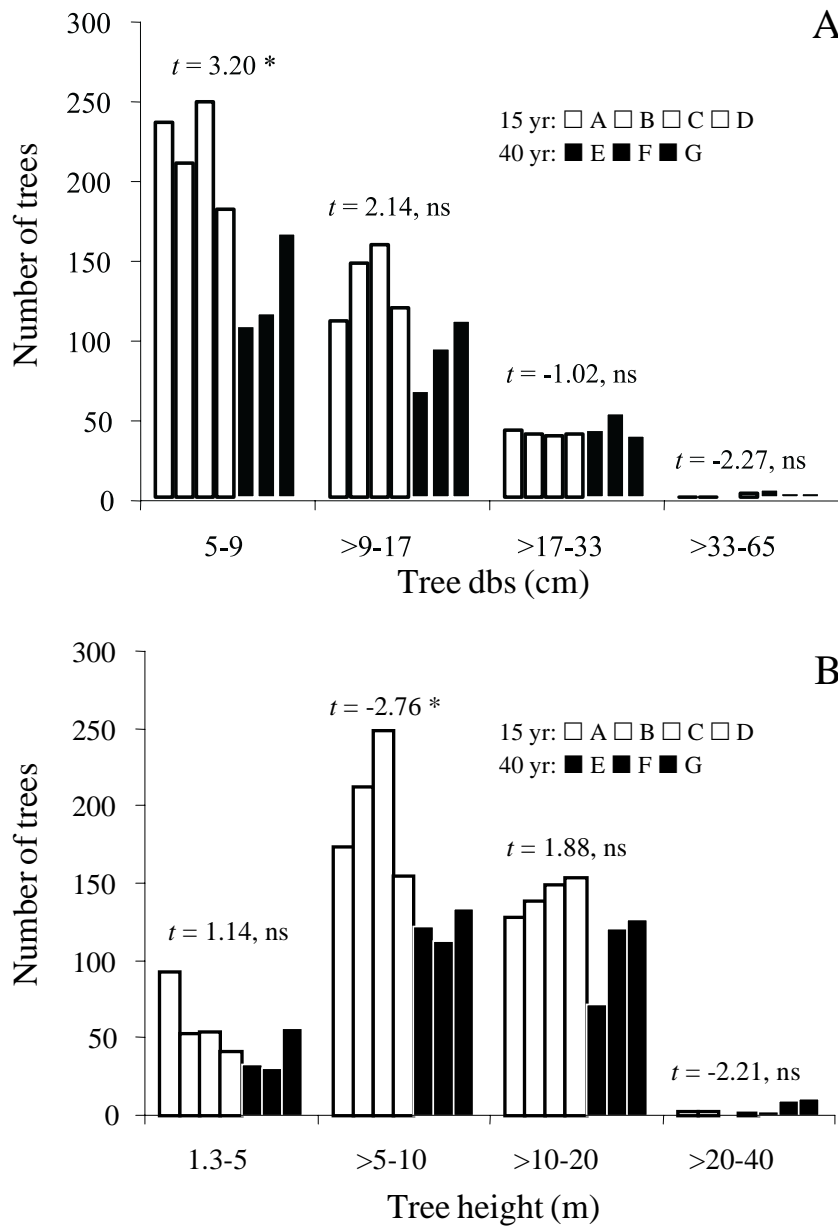

Figure 2. Distribution of the number of trees per diameter (A) and height (B) classes in the seven $1,125 \mathrm{~m}^{2}$ sample plots of secondary forest (A-G) in Itambé do Mato Dentro, southeastern Brazil. For each class of diameter and height, the distributions of the number of trees per plot are compared between the two stand ages (15- and 40-year-old) with twosample $t$ test.
A was not significant because the $95 \%$ confidence intervals for mean $J$ ' of the two series overlap throughout the distribution (0.825-0.826 and 0.823-0.833 at 14 subplots for the younger and older stands, respectively). As the Shannon diversity index is influenced by both the number of species and species evenness, the $H^{\prime}$ - area curves (figure 3C) naturally compromised the richnessand $J$ '-area curves. The younger stand had significantly higher $H^{\prime}$ values at smaller areas ( $\leq 8$ subplots) but were surpassed by the older stand at the sample size of 14 subplots, where $H^{\prime}$ had $95 \%$ confidence intervals of 4.04-4.05 and 4.06-4.13 for the younger and older stands, respectively. Despite this, the Hutcheson's $t$ test did not yield a significant difference between the stands's $H^{\prime}$ (table 2).

The eigenvalues produced by DCA (axis $1=0.305$, axis $2=0.157$ ) were small, indicating 'short' gradients, i.e. most species occurred throughout the gradients, varying essentially in their abundances (ter Braak 1995). The two stand ages appeared as two very distinct subplot clusters in the DCA biplot (figure 4A). In addition, subplots of different plots were generally mixed within their respective age cluster, denoting little to none interplot discrimination. Plot D was the only exception, as its subplots were more discriminated from those of plots $\mathrm{A}, \mathrm{B}$ and $\mathrm{C}$.

The DCA ordination of species (figure 4A) strongly agrees with the differences found between stand ages for tree frequency and volume of particular species (table 4). Of the 47 species used in the DCA, 24 were significantly more frequent in either the younger or the older stand, and 11 out of these 24 species also differed in the distribution of volumes in the 15- and 40-year-old plots. The same 24 species are placed accordingly on 
Table 3. Tree species sampled on seven 1,125 $\mathrm{m}^{2}$ plots of secondary forest in Itambé do Mato Dentro, south-eastern Brazil, followed by brackets containing their classification into species guilds and the number of individuals recorded in the 15 - and 40-year-old plots $\left(\mathrm{N}_{15}\right.$ and $\left.\mathrm{N}_{40}\right)$, respectively. Regeneration guilds: $\mathrm{PI}=$ pioneer, $\mathrm{LD}=$ light-demanding, $\mathrm{ST}=$ shade-tolerant. Stratification guilds: $\mathrm{L}=$ large, $\mathrm{M}$, medium, $\mathrm{S}=$ small. Dispersion guilds: Ane $=$ anemochorous, Aut = autochorous, Zoo $=$ zoochorous. Species are given per family and listed in alphabetical order.

\begin{tabular}{|c|c|c|c|}
\hline Families/Species & Guilds & $\mathrm{N}_{15}$ & $\mathrm{~N}_{4 \mathrm{C}}$ \\
\hline \multicolumn{4}{|l|}{ ACHARIACEAE } \\
\hline Carpotroche brasiliensis (Raddi) A.Gray & ST-L-Zoo & 3 & 8 \\
\hline \multicolumn{4}{|l|}{ ANACARDIACEAE } \\
\hline Astronium fraxinifolium Schott & LD-L-Ane & 96 & 3 \\
\hline Astronium graveolens Jacquin & ST-L-Ane & 3 & 7 \\
\hline Tapirira obtusa (Benth.) Mitchell & LD-L-Zoo & 2 & 0 \\
\hline Thyrsodium spruceanum Salzm. & LD-L-Zoo & 13 & 10 \\
\hline \multicolumn{4}{|l|}{ ANNONACEAE } \\
\hline Anaxagorea phaeocarpa Mart. & ST-L-Zoo & 16 & 12 \\
\hline Duguetia lanceolata A. St.-Hil. & ST-L-Zoo & 9 & 2 \\
\hline Guatteria nigrescens Mart. & ST-M-Zoo & 1 & 0 \\
\hline Guatteria pogonopus Mart. & LD-L-Zoo & 1 & 4 \\
\hline Guatteria villosissima A. St.-Hil. & LD-L-Zoo & 34 & 5 \\
\hline Rollinia laurifolia Schltdl. & LD-L-Zoo & 2 & 0 \\
\hline Xylopia brasiliensis Sprengel & ST-L-Zoo & 9 & 0 \\
\hline Xylopia sericea A. St.-Hil. & LD-M-Zoo & 39 & 14 \\
\hline \multicolumn{4}{|l|}{ APOCYNACEAE } \\
\hline Aspidosperma parvifolium A. DC. & LD-L-Ane & 1 & 1 \\
\hline Aspidosperma ramiflorum Müll. Arg. & LD-L-Ane & 0 & 1 \\
\hline Aspidosperma spruceanum Benth. & LD-L-Ane & 2 & 0 \\
\hline Himatanthus lancifolius (Müll. Arg.) Woodson & LD-M-Ane & 2 & 1 \\
\hline Malouetia arborea (Vell.) Miers & LD-L-Ane & 1 & 1 \\
\hline Tabernaemontana hystrix (Steud.) A. DC. & LD-M-Zoo & 1 & 0 \\
\hline \multicolumn{4}{|l|}{ AQUIFOLIACEAE } \\
\hline Ilex cerasifolia Reissek & ST-M-Zoo & 6 & 13 \\
\hline \multicolumn{4}{|l|}{ ARALIACEAE } \\
\hline Dendropanax cuneatus (DC.) Decne \& Planchon & ST-M-Zoo & 0 & 4 \\
\hline Schefflera morototoni (Aublet) Maguire et al. & LD-L-Zoo & 9 & 2 \\
\hline \multicolumn{4}{|l|}{ ARECACEAE } \\
\hline Bactris acanthocarpa Mart. & ST-S-Zoo & 0 & 1 \\
\hline Polyandrococos caudescens (Mart.) Barb. Rodr. & LD-L-Zoo & 1 & 0 \\
\hline Syagrus romanzoffiana (Cham.) Glassman & LD-L-Zoo & 13 & 2 \\
\hline \multicolumn{4}{|l|}{ ASTERACEAE } \\
\hline Eremanthus incanus (Less.) Less. & PI-M-Ane & 6 & 0 \\
\hline Piptocarpha macropoda Baker & LD-M-Ane & 9 & 0 \\
\hline \multicolumn{4}{|l|}{ BIGNONIACEAE } \\
\hline Jacaranda macrantha Cham. & LD-L-Ane & 3 & 0 \\
\hline Jacaranda puberula Cham. & LD-L-Ane & 5 & 3 \\
\hline Sparattosperma leucanthum (Vell.) K. Schum. & LD-L-Ane & 10 & 1 \\
\hline Tabebuia vellosoi Toledo & ST-L-Ane & 1 & 1 \\
\hline Zeyheria tuberculosa (Vell.) Bureau & LD-L-Ane & 1 & 1 \\
\hline \multicolumn{4}{|l|}{ BORAGINACEAE } \\
\hline Cordia ecalyculata Vell. & LD-L-Zoo & 1 & 0 \\
\hline Cordia magnoliifolia Cham. & ST-M-Zoo & 0 & 1 \\
\hline Cordia sellowiana Cham. & LD-L-Zoo & 1 & 0 \\
\hline \multicolumn{4}{|l|}{ BURSERACEAE } \\
\hline Protium brasiliense (Sprengel) Engler & ST-M-Zoo & 1 & 0 \\
\hline Protium heptaphyllum (Aublet) Marchand & LD-L-Zoo & 15 & 10 \\
\hline
\end{tabular}




\begin{tabular}{|c|c|c|c|}
\hline Families/Species & Guilds & $\mathrm{N}_{15}$ & $\mathrm{~N}_{40}$ \\
\hline \multicolumn{4}{|l|}{ BURSERACEAE } \\
\hline Protium spruceanum (Benth.) Engler & ST-L-Zoo & 9 & 8 \\
\hline Protium warmingianum March. & ST-L-Zoo & 0 & 7 \\
\hline \multicolumn{4}{|l|}{ CELASTRACEAE } \\
\hline Maytenus glazioviana Loes. & ST-M-Zoo & 0 & 1 \\
\hline Maytenus robusta Reissek & ST-M-Zoo & 4 & 0 \\
\hline Maytenus salicifolia Reissek & ST-M-Zoo & 1 & 2 \\
\hline \multicolumn{4}{|l|}{ CHRYSOBALANACEAE } \\
\hline Couepia meridionalis Prance & LD-L-Zoo & 3 & 1 \\
\hline \multicolumn{4}{|l|}{ CHRYSOBALANACEAE } \\
\hline Licania hypoleuca Benth. & LD-L-Zoo & 71 & 30 \\
\hline Licania octandra (Hoffmgg.) Kuntze & LD-L-Zoo & 3 & 0 \\
\hline \multicolumn{4}{|l|}{ CLETHRACEAE } \\
\hline Clethra scabra Pers. & LD-M-Ane & 1 & 0 \\
\hline \multicolumn{4}{|l|}{ CLUSIACEAE } \\
\hline Calophyllum brasiliense Cambess. & ST-L-Zoo & 4 & 3 \\
\hline Kielmeyera lathrophyton Saddi & LD-M-Ane & 2 & 0 \\
\hline Tovomitopsis saldanhae Engler & ST-L-Zoo & 0 & 3 \\
\hline Vismia guianensis (Aublet) Pers. & PI-M-Zoo & 2 & 0 \\
\hline \multicolumn{4}{|l|}{ COMBRETACEAE } \\
\hline Terminalia glabrescens Mart. & LD-L-Ane & 1 & 1 \\
\hline \multicolumn{4}{|l|}{ CYATHEACEAE } \\
\hline Cyathea corcovadensis (Raddi) Domin & ST-M-Ane & 0 & 1 \\
\hline Cyathea delgadii Sternb. & ST-S-Ane & 0 & 2 \\
\hline Cyathea leucofolis Domin & ST-S-Ane & 0 & 1 \\
\hline \multicolumn{4}{|l|}{ DICHAPETALACEAE } \\
\hline Stephanopodium engleri Baillon & ST-M-Zoo & 2 & 0 \\
\hline \multicolumn{4}{|l|}{ EBENACEAE } \\
\hline Diospyros hispida A.DC. & LD-L-Zoo & 1 & 0 \\
\hline \multicolumn{4}{|l|}{ ERYTHROXYLACEAE } \\
\hline Erythroxylum citrifolium A. St.-Hil. & ST-M-Zoo & 1 & 0 \\
\hline Erythroxylum pelleterianum A. St.-Hil. & ST-S-Zoo & 10 & 3 \\
\hline \multicolumn{4}{|l|}{ EUPHORBIACEAE } \\
\hline Actinostemon klotzschii (Didrichs) Pax & ST-S-Aut & 1 & 0 \\
\hline Aparisthmium cordatum (Juss.) Baillon & LD-L-Aut & 0 & 2 \\
\hline Chaetocarpus echinocarpus (Baillon) Ducke & LD-M-Aut & 26 & 6 \\
\hline Croton floribundus Sprengel & PI-L-Aut & 1 & 0 \\
\hline Gymnanthes concolor (Sprengel) Müll. Arg. & LD-M-Aut & 0 & 17 \\
\hline Mabea fistulifera Mart. & PI-M-Aut & 69 & 39 \\
\hline Maprounea guianensis Aublet & LD-M-Aut & 1 & 0 \\
\hline Pera glabrata (Schott) Poepp. & LD-L-Zoo & 99 & 18 \\
\hline Pogonophora schomburgkiana Miers & ST-L-Aut & 52 & 16 \\
\hline Sapium glandulosum (L.) Morong & LD-L-Zoo & 0 & 2 \\
\hline \multicolumn{4}{|l|}{ FABACEAE } \\
\hline Acacia polyphylla DC. & LD-L-Ane & 0 & 1 \\
\hline Albizia polycephala (Benth.) Killip & LD-L-Ane & 0 & 4 \\
\hline Anadenanthera colubrina (Vell.) Brenan & PI-L-Ane & 2 & 2 \\
\hline Andira fraxinifolia Benth. & ST-L-Zoo & 0 & 2 \\
\hline Apuleia leiocarpa (Vogel) Macbr. & LD-L-Ane & 90 & 99 \\
\hline Cassia ferruginea (Schrad.) Schrad. & LD-L-Zoo & 3 & 3 \\
\hline Copaifera langsdorffii Desf. & LD-L-Zoo & 18 & 5 \\
\hline Dalbergia frutescens (Vell.) Britton & LD-L-Ane & 1 & 2 \\
\hline
\end{tabular}


continuation

\begin{tabular}{|c|c|c|c|}
\hline Families/Species & Guilds & $\mathrm{N}_{15}$ & $\mathrm{~N}_{40}$ \\
\hline \multicolumn{4}{|l|}{ FABACEAE } \\
\hline Dalbergia nigra (Vell.) Fr. Allem. & LD-L-Ane & 37 & 13 \\
\hline Dalbergia villosa (Benth.) Benth. & LD-L-Ane & 20 & 2 \\
\hline Hymenolobium janeirense Kuhlman & LD-L-Ane & 2 & 2 \\
\hline Inga marginata Willd. & ST-M-Zoo & 2 & 0 \\
\hline Inga striata Benth. & LD-L-Zoo & 2 & 6 \\
\hline Inga vera Willd. & LD-L-Zoo & 0 & 1 \\
\hline Machaerium acutifolium Vogel & LD-L-Ane & 2 & 1 \\
\hline Machaerium brasiliense Vogel & LD-L-Ane & 5 & 0 \\
\hline Machaerium dimorphandrum Hoehne & LD-L-Ane & 3 & 0 \\
\hline Machaerium lanceolatum (Vell.) Macbr. & LD-L-Ane & 0 & 1 \\
\hline Machaerium stipitatum (DC.) Vogel & LD-L-Ane & 3 & 1 \\
\hline Melanoxylon brauna Schott & ST-L-Ane & 8 & 11 \\
\hline Piptadenia gonoacantha (Mart.) Macbr. & PI-L-Ane & 0 & 2 \\
\hline Plathymenia reticulata Benth. & LD-L-Ane & 8 & 3 \\
\hline Platypodium elegans Vogel & LD-L-Ane & 17 & 5 \\
\hline Pseudopiptadenia contorta (DC.) Lewis \& H.C. Lima & LD-L-Ane & 1 & 0 \\
\hline Sclerolobium rugosum Mart. & LD-L-Ane & 5 & 2 \\
\hline Senna multijuga (L.C. Rich.) Irwin \& Barneby & LD-L-Zoo & 2 & 0 \\
\hline Swartzia acutifolia Vogel & ST-L-Zoo & 6 & 0 \\
\hline Swartzia apetala Raddi & ST-L-Zoo & 2 & 0 \\
\hline Swartzia flaemingii Vogel & LD-L-Zoo & 0 & 1 \\
\hline Swartzia macrostachya Benth. & LD-L-Zoo & 16 & 0 \\
\hline Swartzia multijuga Hayne & LD-L-Zoo & 1 & 1 \\
\hline Swartzia myrtifolia (Schott) Cowan & LD-L-Zoo & 0 & 1 \\
\hline Swartzia polyphylla DC. & LD-L-Zoo & 7 & 1 \\
\hline Sweetia fruticosa Sprengel & LD-L-Ane & 0 & 3 \\
\hline Tachigali paratyensis (Vell.) H.C. Lima & ST-L-Ane & 1 & 1 \\
\hline Zygia latifolia (L.) Fawc. \& Rendle & ST-L-Aut & 15 & 2 \\
\hline \multicolumn{4}{|l|}{ LAURACEAE } \\
\hline Aniba firmula (Nees \& Mart.) Mez & LD-L-Zoo & 4 & 1 \\
\hline Endlicheria glomerata $\mathrm{Mez}$ & ST-M-Zoo & 5 & 4 \\
\hline Nectandra megapotamica (Sprengel) Mez & LD-L-Zoo & 3 & 0 \\
\hline Nectandra oppositifolia Nees & ST-L-Zoo & 1 & 1 \\
\hline Nectandra reticulata (Ruiz \& Pavón) Mez & ST-L-Zoo & 1 & 0 \\
\hline Nectandra warmingii Meisner & ST-L-Zoo & 0 & 3 \\
\hline Ocotea corymbosa (Meisner) Mez & LD-L-Zoo & 7 & 6 \\
\hline Ocotea dispersa (Nees) Mez & LD-L-Zoo & 3 & 0 \\
\hline Ocotea divaricata (Nees) $\mathrm{Mez}$ & ST-L-Zoo & 1 & 1 \\
\hline Ocotea laxa (Nees) Mez & ST-M-Zoo & 0 & 1 \\
\hline Ocotea odorifera (Vell.) Rohwer & ST-L-Zoo & 0 & 2 \\
\hline Ocotea velutina (Nees) Rohwer & LD-L-Zoo & 4 & 6 \\
\hline Urbanodendron verrucosum (Nees) Mez & ST-L-Zoo & 3 & 3 \\
\hline \multicolumn{4}{|l|}{ LECYTHIDACEAE } \\
\hline Cariniana estrellensis (Raddi) Kuntze & LD-L-Ane & 0 & 1 \\
\hline Lecythis lanceolata Poiret & ST-M-Zoo & 0 & 1 \\
\hline \multicolumn{4}{|l|}{ MALPIGHIACEAE } \\
\hline Byrsonima sericea DC. & LD-L-Zoo & 32 & 1 \\
\hline Heteropterys byrsonimifolia A. Juss. & LD-M-Ane & 2 & 0 \\
\hline \multicolumn{4}{|l|}{ MALVACEAE } \\
\hline Eriotheca candolleana (K. Schum.) A. Robyns & LD-L-Ane & 1 & 1 \\
\hline Luehea divaricata Mart. \& Zucc. & LD-L-Ane & 4 & 4 \\
\hline
\end{tabular}




\begin{tabular}{|c|c|c|c|}
\hline Families/Species & Guilds & $\mathrm{N}_{15}$ & $\mathrm{~N}_{40}$ \\
\hline \multicolumn{4}{|l|}{ MALVACEAE } \\
\hline Luehea grandiflora Mart. \& Zucc. & LD-L-Ane & 0 & 5 \\
\hline \multicolumn{4}{|l|}{ MELASTOMATACEAE } \\
\hline Miconia calvescens DC. & ST-M-Zoo & 0 & 4 \\
\hline Miconia fasciculata Gardner & ST-M-Zoo & 0 & 1 \\
\hline Miconia trianae Cogn. & LD-M-Zoo & 4 & 0 \\
\hline Tibouchina granulosa Cogn. & LD-M-Ane & 0 & 2 \\
\hline \multicolumn{4}{|l|}{ MELIACEAE } \\
\hline Guarea guidonia (L.) Sleumer & ST-M-Zoo & 1 & 1 \\
\hline Guarea kunthiana A. Juss. & ST-L-Zoo & 2 & 0 \\
\hline Trichilia hirta L. & LD-L-Zoo & 2 & 0 \\
\hline Trichilia pallens C.DC. & ST-M-Zoo & 2 & 0 \\
\hline Trichilia pallida Swartz & ST-M-Zoo & 6 & 24 \\
\hline \multicolumn{4}{|l|}{ MORACEAE } \\
\hline Brosimum guianense (Aublet) Huber & LD-L-Zoo & 4 & 2 \\
\hline Brosimum lactescens (S. Moore) C.C. Berg. & LD-L-Zoo & 7 & 11 \\
\hline Ficus gomelleira Kunth \& Bouché & ST-L-Zoo & 1 & 0 \\
\hline Sorocea guilleminiana Gaud. & ST-M-Zoo & 4 & 1 \\
\hline \multicolumn{4}{|l|}{ MYRTACEAE } \\
\hline Calyptranthes clusiifolia (Miq.) O. Berg & ST-M-Zoo & 7 & 3 \\
\hline Campomanesia dichotoma (O. Berg) Mattos & LD-M-Zoo & 17 & 9 \\
\hline Campomanesia xanthocarpa O. Berg & LD-M-Zoo & 0 & 1 \\
\hline Eugenia cuprea (O. Berg) Nied. & LD-L-Zoo & 0 & 1 \\
\hline Eugenia florida DC. & ST-M-Zoo & 13 & 17 \\
\hline Eugenia neoglomerata Sobral & ST-L-Zoo & 0 & 4 \\
\hline Eugenia neolanceolata Sobral & ST-L-Zoo & 32 & 13 \\
\hline Gomidesia anacardiifolia (Gardner) O. Berg & ST-M-Zoo & 10 & 9 \\
\hline Marlierea warmingiana Kiaersk. & ST-L-Zoo & 16 & 1 \\
\hline Myrcia detergens Miq. & LD-L-Zoo & 12 & 5 \\
\hline Myrcia eriopus DC. & ST-M-Zoo & 0 & 1 \\
\hline Myrcia rufula Miq. & LD-M-Zoo & 0 & 2 \\
\hline Myrciaria glomerata $\mathrm{O}$. Berg & ST-S-Zoo & 4 & 1 \\
\hline Myrciaria tenella (DC.) O. Berg & ST-M-Zoo & 0 & 7 \\
\hline Pimenta pseudocaryophyllus (Gomes) Landrum & LD-M-Zoo & 88 & 1 \\
\hline Plinia cauliflora (Mart.) Kausel & ST-L-Zoo & 6 & 1 \\
\hline Psidium cattleianum Sabine & ST-L-Zoo & 1 & 0 \\
\hline Psidium robustum $\mathrm{O} . \mathrm{Berg}$ & LD-M-Zoo & 3 & 0 \\
\hline Psidium rufum Mart. & LD-M-Zoo & 1 & 7 \\
\hline \multicolumn{4}{|l|}{ NYCTAGINACEAE } \\
\hline Guapira hirsuta (Choisy) Lundell & ST-M-Zoo & 3 & 1 \\
\hline Guapira opposita (Vell.) Reitz & ST-M-Zoo & 9 & 3 \\
\hline \multicolumn{4}{|l|}{ OCHNACEAE } \\
\hline Ouratea parviflora Baillon & ST-M-Zoo & 2 & 0 \\
\hline \multicolumn{4}{|l|}{ OLACACEAE } \\
\hline Schoepfia brasiliensis A. DC. & ST-M-Zoo & 2 & 4 \\
\hline \multicolumn{4}{|l|}{ PHYLLANTHACEAE } \\
\hline Savia dictyocarpa Müll. Arg. & ST-L-Aut & 6 & 7 \\
\hline \multicolumn{4}{|l|}{ PICRAMNIACEAE } \\
\hline Picramnia parvifolia Engler & ST-M-Zoo & 1 & 0 \\
\hline \multicolumn{4}{|l|}{ POACEAE } \\
\hline Guadua angustifolia Kunth & LD-L-Ane & 1 & 0 \\
\hline
\end{tabular}


continuation

\begin{tabular}{|c|c|c|c|}
\hline Families/Species & Guilds & $\mathrm{N}_{15}$ & $\mathrm{~N}_{40}$ \\
\hline \multicolumn{4}{|l|}{ PROTEACEAE } \\
\hline Euplassa incana (Klotzsch) Johnston & LD-L-Zoo & 0 & 1 \\
\hline Euplassa legalis (Vell.) Johnston & LD-L-Zoo & 0 & 1 \\
\hline \multicolumn{4}{|l|}{ RUBIACEAE } \\
\hline Amaioua guianensis Aublet & ST-M-Zoo & 12 & 7 \\
\hline Bathysa nicholsonii K. Schum. & ST-M-Ane & 8 & 54 \\
\hline Chomelia catharinae (Smith \& Downs) Steyerm. & ST-M-Zoo & 0 & 2 \\
\hline Genipa infundibuliformis Zappi \& Semir & LD-L-Zoo & 1 & 1 \\
\hline Guettarda uruguensis Cham. \& Schltdl. & LD-L-Zoo & 3 & 2 \\
\hline Ixora warmingii Müll. Arg. & ST-M-Zoo & 4 & 0 \\
\hline Psychotria carthagenensis Jacquin & ST-S-Zoo & 0 & 1 \\
\hline Psychotria vellosiana Benth. & LD-M-Zoo & 0 & 1 \\
\hline Rudgea jasminoides (Cham.) Müll. Arg. & ST-M-Zoo & 1 & 1 \\
\hline Tocoyena sellowiana (Cham. \& Schltdl.) K. Schum. & ST-M-Zoo & 1 & 1 \\
\hline Warszewiczia longistaminea K. Schum. & LD-L-Ane & 1 & 0 \\
\hline \multicolumn{4}{|l|}{ RUTACEAE } \\
\hline Esenbeckia febrifuga (A. St.-Hil.) A. Juss. & ST-M-Aut & 0 & 2 \\
\hline Pilocarpus giganteus Engler & ST-L-Aut & 23 & 6 \\
\hline Zanthoxylum rhoifolium Lam. & LD-L-Zoo & 0 & 1 \\
\hline Zanthoxylum tingoassuiba A. St.-Hil. & LD-L-Zoo & 0 & 1 \\
\hline \multicolumn{4}{|l|}{ SALICACEAE } \\
\hline Casearia decandra Jacquin & ST-M-Zoo & 5 & 0 \\
\hline Casearia ulmifolia Vahl & LD-L-Zoo & 4 & 5 \\
\hline Xylosma ciliatifolia (Clos) Eichler & LD-M-Zoo & 34 & 2 \\
\hline \multicolumn{4}{|l|}{ SAPINDACEAE } \\
\hline Allophylus edulis (A. St.-Hil.) Radlk. & ST-M-Zoo & 5 & 1 \\
\hline Cupania emarginata Cambess. & LD-L-Zoo & 41 & 6 \\
\hline Cupania oblongifolia Mart. & LD-L-Zoo & 1 & 0 \\
\hline Cupania vernalis Cambess. & LD-L-Zoo & 4 & 4 \\
\hline Dilodendron elegans (Radlk.) Gentry \& Steyerm. & LD-L-Zoo & 1 & 0 \\
\hline Matayba elaeagnoides Radlk. & ST-L-Zoo & 16 & 3 \\
\hline Matayba guianensis Aublet & LD-M-Zoo & 19 & 0 \\
\hline Matayba juglandifolia (Cambess.) Radlk. & ST-L-Zoo & 4 & 1 \\
\hline Toulicia laevigata Radlk. & LD-M-Ane & 81 & 1 \\
\hline \multicolumn{4}{|l|}{ SAPOTACEAE } \\
\hline Micropholis gardneriana (A. DC.) Pierre & LD-L-Zoo & 3 & 4 \\
\hline \multicolumn{4}{|l|}{ SIPARUNACEAE } \\
\hline Siparuna guianensis Aublet & ST-S-Zoo & 23 & 62 \\
\hline \multicolumn{4}{|l|}{ SOLANACEAE } \\
\hline Solanum leucodendron Sendt. & LD-L-Zoo & 0 & 1 \\
\hline \multicolumn{4}{|l|}{ URTICACEAE } \\
\hline Cecropia hololeuca Miq. & PI-L-Zoo & 0 & 19 \\
\hline Pourouma guianensis Aublet & PI-L-Zoo & 1 & 0 \\
\hline \multicolumn{4}{|l|}{ VERBENACEAE } \\
\hline Aloysia virgata (Ruiz \& Pavón) A. Juss. & PI-S-Ane & 8 & 2 \\
\hline \multicolumn{4}{|l|}{ VOCHYSIACEAE } \\
\hline Vochysia magnifica Warm. & ST-L-Ane & 1 & 0 \\
\hline
\end{tabular}


either the left or right sides of the DCA biplot (figure 4B), corresponding to the younger and older stands, respectively. Apuleia leiocarpa, Bathysa nicholsonii, Brosimum lactescens, Cecropia hololeuca, Eugenia neoglomerata, Siparuna guianensis and Trichilia pallida were significantly more abundant in the older stand in terms of both tree frequency and volume.

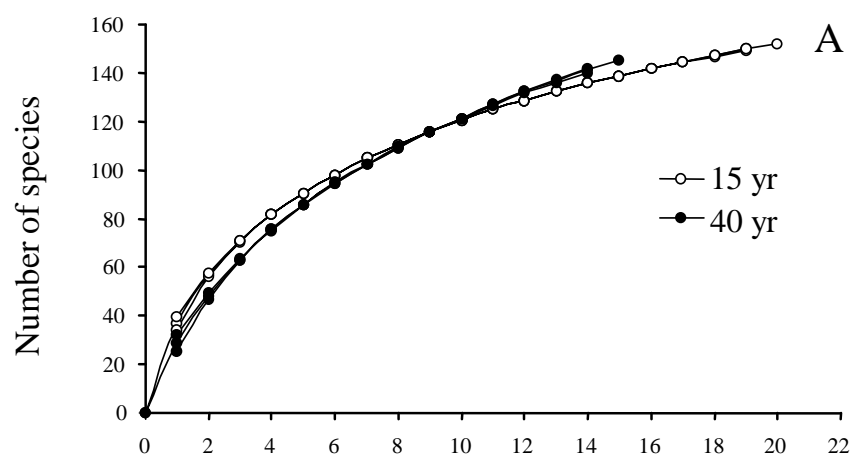

Number of subplots

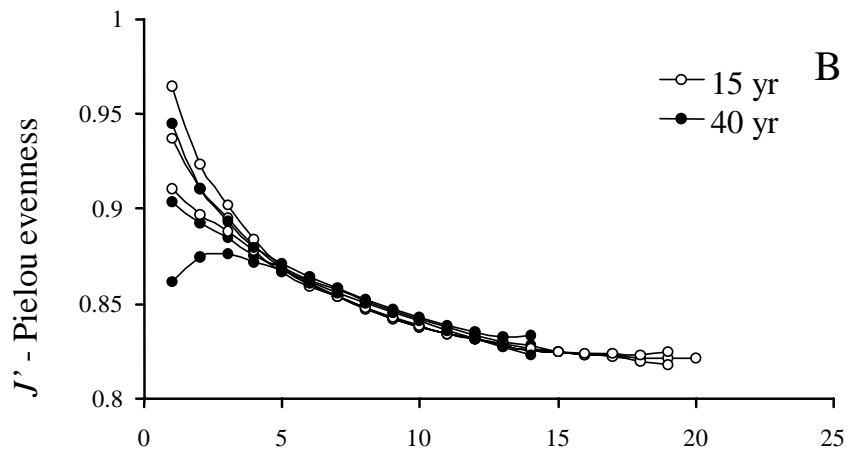

Number of subplots

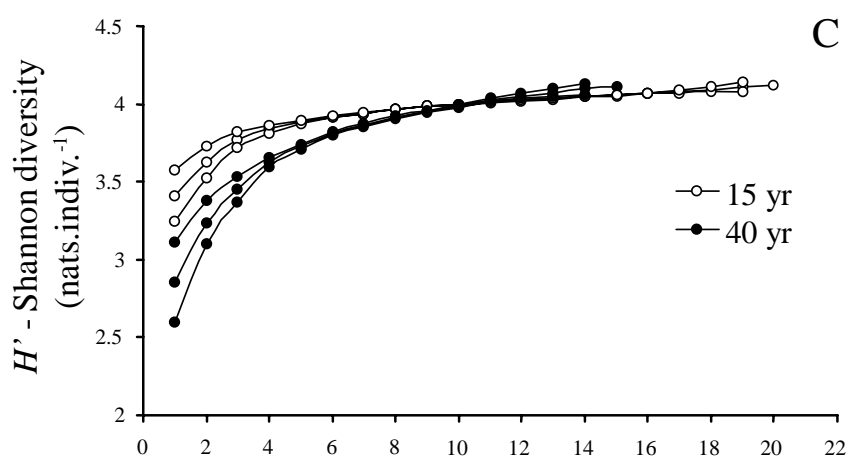

Number of subplots

Figure 3. Progression of three measures of species diversity with increasing sample area for the two stand ages, 15- and 40-year-old, of the secondary forest surveyed in Itambé do Mato Dentro, south-eastern Brazil: (A) number of species, (B) Pielou evenness and (C) Shannon diversity. Curves for each stand age are means $\pm 95 \%$ confidence intervals obtained from sub-sampling all possible combinations of subplots.
Guatteria pogonopus, Eugenia florida, Ilex cerasifolia, Inga striata, Melanoxylon brauna were also significantly more abundant in the older stand but in terms of only tree frequency. All of the above species are also concentrated on the right side of the DCA biplot. Astronium fraxinifolium, Byrsonima sericea, Pimenta pseudocaryophyllus, and Toulicia laevigata were


Figure 4. Detrended correspondence analysis. Biplots showing the ordination in the first two axes of (A) 35 subplots of secondary forest surveyed in Itambé do Mato Dentro, south-eastern Brazil and (B) 47 tree species. The seven sample plots (A-G) are indicated by different symbols: open symbols $=15$-year-old stand ; closed symbols $=40$-year-old stand. Species names are abbreviated; full names in table 4 . 
Table 4. Tree species abundances in the two stands of secondary forest in Itambé do Mato Dentro, south-eastern Brazil. Total number of trees and mean volume per plot in the two stand ages (15- and 40-year-old plots) of the 47 species used in CCA. Full species names are given for the abbreviations used in CCA. The numbers of trees are compared between stand ages with chisquare tests (expected values within brackets are based on the stands' totals) and the distributions of volumes are compared with $t$ tests. $*=P<0.05 ; * *=P<0.01 ; * * *=P<0.005 ; * * * *=P<0.001 ;$ ns $=$ non significant.

\begin{tabular}{|c|c|c|c|c|c|c|c|c|c|}
\hline \multirow{2}{*}{$\begin{array}{l}\text { Tree Specie } \\
\text { Abbrev. }\end{array}$} & \multirow{2}{*}{ Full names } & \multicolumn{2}{|c|}{ Number of trees } & \multirow{2}{*}{\multicolumn{2}{|c|}{$\begin{array}{c}\chi^{2} \\
\text { tests }\end{array}$}} & \multicolumn{2}{|c|}{ Mean volume $\left(\mathrm{m}^{3}\right)$} & \multirow{2}{*}{\multicolumn{2}{|c|}{$t$ tests }} \\
\hline & & $15 \mathrm{yr}$ & $40 \mathrm{yr}$ & & & $15 \mathrm{yr}$ & $40 \mathrm{yr}$ & & \\
\hline Aloy vir & Aloysia virgata & $8 \quad(6.6)$ & $2(3.4)$ & 1.19 & $\mathrm{~ns}$ & 0.748 & 0.175 & $0.89 \mathrm{r}$ & $\mathrm{ns}$ \\
\hline Anad col & Anadenanthera colubrina & $2 \quad(2.6)$ & $2(1.4)$ & 0.51 & ns & 0.006 & 0.751 & $1.98 \mathrm{r}$ & \\
\hline Anax pha & Anaxagorea phaeocarpa & $16(18.5)$ & $12(9.5)$ & 0.89 & $\mathrm{~ns}$ & 0.124 & 0.555 & $1.75 \mathrm{r}$ & $\mathrm{ns}$ \\
\hline Apul lei & Apuleia leiocarpa & $90(124.7)$ & $99(64.3)$ & 28.09 & $* * * *$ & 2.133 & 7.122 & $3.02 *$ & * \\
\hline Astr fra & Astronium fraxinifolium & $96(65.3)$ & $3(33.7)$ & 42.84 & $* * * *$ & 0.596 & 0.037 & $2.71 *$ & * \\
\hline Bath nic & Bathysa nicholsonii & $8(40.9)$ & $54(21.1)$ & 77.02 & $* * * *$ & 0.075 & 0.793 & $4.81 *$ & $* *$ \\
\hline Bros lac & Brosimum lactescens & 7 (11.9) & $11(6.1)$ & 5.56 & $*$ & 0.050 & 0.210 & $2.97 *$ & \\
\hline Byrs ser & Byrsonima sericea & $32(21.8)$ & $1(11.2)$ & 14.60 & $* * * *$ & 1.384 & 0.098 & $3.60 *$ & $*$ \\
\hline Caly clu & Calyptranthes clusiifolia & $7 \quad(6.6)$ & $3(3.4)$ & 0.24 & $\mathrm{~ns}$ & 0.151 & 0.204 & $0.19 \mathrm{r}$ & $\mathrm{ns}$ \\
\hline Camp dic & Campomanesia dichotoma & $17(17.2)$ & $9(8.8)$ & 0.04 & $\mathrm{~ns}$ & 0.291 & 0.624 & $0.62 \mathrm{r}$ & $\mathrm{ns}$ \\
\hline Cecr hol & Cecropia hololeuca & $0(12.5)$ & $19(6.5)$ & 35.95 & $* * * *$ & 0.000 & 1.243 & $2.82 *$ & * \\
\hline Chae ech & Chaetocarpus echinocarpus & $26(21.1)$ & $6(10.9)$ & 3.58 & $\mathrm{~ns}$ & 0.458 & 0.043 & $1.65 \mathrm{r}$ & $\mathrm{ns}$ \\
\hline Copa lan & Copaifera langsdorffii & $18(15.2)$ & $5(7.8)$ & 1.77 & $\mathrm{~ns}$ & 0.222 & 0.284 & $0.20 \mathrm{r}$ & $\mathrm{ns}$ \\
\hline Cupa ema & Cupania emarginata & $41 \quad(31)$ & $6(16)$ & 9.79 & $* * *$ & 0.612 & 0.341 & $0.78 \mathrm{r}$ & $\mathrm{ns}$ \\
\hline Dalb nig & Dalbergia nigra & $37 \quad(33)$ & $13 \quad(17)$ & 1.57 & $\mathrm{~ns}$ & 0.385 & 0.372 & $0.05 \mathrm{r}$ & $\mathrm{ns}$ \\
\hline Dalb vil & Dalbergia villosa & $20(14.5)$ & $2(7.5)$ & 6.50 & $*$ & 0.355 & 0.038 & $1.43 \mathrm{r}$ & $\mathrm{ns}$ \\
\hline Dugu lan & Duguetia lanceolata & $9 \quad(7.3)$ & $2(3.7)$ & 1.56 & $\mathrm{~ns}$ & 0.442 & 0.071 & $1.80 \mathrm{r}$ & $\mathrm{ns}$ \\
\hline Euge flo & Eugenia florida & $13(19.8)$ & $17(10.2)$ & 6.56 & $*$ & 0.070 & 0.312 & $1.24 \mathrm{r}$ & $\mathrm{ns}$ \\
\hline Euge nel & Eugenia neoglomerata & $0 \quad(2.6)$ & $4(1.4)$ & 7.09 & $* *$ & 0.307 & 1.485 & $5.86 *$ & $* *$ \\
\hline Gomi ana & Gomidesia anacardiifolia & $10(12.5)$ & $9(6.5)$ & 1.37 & $\mathrm{~ns}$ & 0.138 & 46 & $0.05 \mathrm{r}$ & $\mathrm{ns}$ \\
\hline Guat pog & Guatteria pogonopиs & $1 \quad(3.3)$ & $4(1.7)$ & 4.27 & $*$ & 0.010 & 16 & $1.93 \mathrm{r}$ & $\mathrm{ns}$ \\
\hline Guat vil & Guatteria villosissima & $34(25.7)$ & $5(13.3)$ & 8.15 & $* * *$ & 0.242 & 0.074 & $1.42 \mathrm{r}$ & $\mathrm{ns}$ \\
\hline Ilex cer & Ilex cerasifolia & $6(12.5)$ & $13(6.5)$ & 9.58 & $* * *$ & 0.023 & 0.215 & $1.68 \mathrm{r}$ & $\mathrm{ns}$ \\
\hline Inga str & Inga striata & $2(5.3)$ & $6(2.7)$ & 5.54 & * & 0.024 & 0.832 & $2.07 \mathrm{r}$ & $\mathrm{ns}$ \\
\hline Lica hyp & Licania hypoleuca & $71(66.6)$ & $30(34.4)$ & 0.92 & $\mathrm{~ns}$ & 1.985 & 1.184 & $1.11 \mathrm{r}$ & $\mathrm{ns}$ \\
\hline Mabe fis & Mabea fistulifera & $69(71.2)$ & $39(36.8)$ & 0.19 & $\mathrm{~ns}$ & 0.942 & 1.249 & $0.54 \mathrm{r}$ & $\mathrm{ns}$ \\
\hline Mata ela & Matayba elaeagnoides & $16(12.5)$ & $3(6.5)$ & 3.13 & $\mathrm{~ns}$ & 0.141 & 0.347 & $0.59 \mathrm{r}$ & $\mathrm{ns}$ \\
\hline Mata gui & Matayba guianensis & $19(12.5)$ & $0(6.5)$ & 10.34 & $* * *$ & 0.314 & 0.000 & $1.38 \mathrm{r}$ & $\mathrm{ns}$ \\
\hline Mela bra & Melanoxylon brauna & $8(12.5)$ & $11(6.5)$ & 4.54 & $*$ & 0.104 & 0.824 & $1.64 \mathrm{r}$ & $\mathrm{ns}$ \\
\hline Pera gla & Pera glabrata & $99(77.2)$ & $18(39.8)$ & 18.40 & $* * * *$ & 4.387 & 1.500 & $2.20 \mathrm{r}$ & $\mathrm{ns}$ \\
\hline Pilo gig & Pilocarpus giganteus & $23(19.1)$ & $6(9.9)$ & 2.53 & $\mathrm{~ns}$ & 0.969 & 0.339 & $0.85 \mathrm{r}$ & $\mathrm{ns}$ \\
\hline Pime pse & Pimenta pseudocaryophyllus & $88(58.7)$ & $1(30.3)$ & 43.41 & $* * * *$ & 2.145 & 0.107 & $3.32 *$ & * \\
\hline Pipt mac & Piptocarpha macropoda & $9 \quad(5.9)$ & $0(3.1)$ & 5.25 & * & 0.358 & 0.000 & $1.14 \mathrm{r}$ & $\mathrm{ns}$ \\
\hline Plat ele & Platypodium elegans & $17(14.5)$ & $5(7.5)$ & 1.46 & $\mathrm{~ns}$ & 0.384 & 0.176 & $0.72 \mathrm{r}$ & $\mathrm{ns}$ \\
\hline Plat ret & Plathymenia reticulata & 8 (7.3) & $3(3.7)$ & 0.42 & $\mathrm{~ns}$ & 0.185 & 0.764 & $0.87 \mathrm{r}$ & $\mathrm{ns}$ \\
\hline Pogo sch & Pogonophora schomburgkiana & $52(44.9)$ & $16(23.1)$ & 3.51 & $\mathrm{~ns}$ & 0.584 & 0.596 & $0.02 \mathrm{r}$ & $\mathrm{ns}$ \\
\hline Prot hep & Protium heptaphyllum & $15(16.5)$ & $10(8.5)$ & 0.36 & $\mathrm{~ns}$ & 0.275 & 1.138 & $1.15 \mathrm{r}$ & $\mathrm{ns}$ \\
\hline Sche mor & Schefflera morototoni & 9 (7.3) & $2(3.7)$ & 1.56 & $\mathrm{~ns}$ & 0.273 & 0.045 & $1.85 \mathrm{r}$ & $\mathrm{ns}$ \\
\hline Scle rug & Sclerolobium rugosum & $5 \quad(4.6)$ & $2(2.4)$ & 0.33 & $\mathrm{~ns}$ & 1.155 & 0.896 & $0.15 \mathrm{r}$ & $\mathrm{ns}$ \\
\hline Sipa gui & Siparuna guianensis & $23(56.1)$ & $62(28.9)$ & 56.78 & $* * * *$ & 0.054 & 0.265 & $3.06 *$ & $*$ \\
\hline Syag rom & Syagrus romanzoffiana & $13 \quad(9.9)$ & $2(5.1)$ & 3.23 & $\mathrm{~ns}$ & 0.325 & 0.161 & $0.68 \mathrm{r}$ & $\mathrm{ns}$ \\
\hline Thyr spr & Thyrsodium spruceanum & $13(15.2)$ & $10(7.8)$ & 0.83 & ns & 0.408 & 0.615 & $0.67 \mathrm{r}$ & $\mathrm{ns}$ \\
\hline Toul lae & Toulicia laevigata & $81(54.1)$ & $1(27.9)$ & 39.81 & $* * * *$ & 0.446 & 0.023 & 2.79 & * \\
\hline Tric pal & Trichilia pallida & $6(19.8)$ & $24(10.2)$ & 27.62 & $* * * *$ & 0.017 & 0.189 & 1.48 & $*$ \\
\hline Xylo cil & Xylosma ciliatifolia & $34(23.7)$ & $2(12.3)$ & 13.44 & $* * * *$ & 0.158 & 0.011 & $1.39 \mathrm{r}$ & $\mathrm{ns}$ \\
\hline Xylo ser & Xylopia sericea & $39 \quad(35)$ & $14 \quad(18)$ & 1.50 & $\mathrm{~ns}$ & 0.776 & 0.548 & $0.50 \mathrm{r}$ & $\mathrm{ns}$ \\
\hline Zygi lat & Zygia latifolia & $15(11.2)$ & $2(5.8)$ & 4.14 & * & 0.228 & 0.071 & $0.72 \mathrm{r}$ & $\mathrm{ns}$ \\
\hline
\end{tabular}


significantly more abundant in the younger stand in terms of both tree frequency and volume. Cupania emarginata, Dalbergia villosa, Guatteria villosissima, Matayba guianensis, Pera glabrata, Piptocarpha macropoda, Xylosma ciliatifolia and Zygia latifolia were also significantly more abundant in the younger stand but in terms of only tree frequency. All of these species are also concentrated on the left side of the DCA biplot.

The two stand communities differed in the proportions of trees per species guild, for regeneration and stratification but not for dispersion (table 5). With respect to expected values for regeneration guilds, lightdemanding trees were more frequent in the younger stand and less frequent in the older stand. Shade-tolerant and pioneer trees followed the opposite pattern, being more frequent in the older stand. With respect to expected values for stratification guilds, only trees of understory species (small size at maturity) were more frequent in the older stand and less frequent in the younger stand.

\section{Discussion}

Numerous studies of tropical forests worldwide have demonstrated that tree species distribution is highly influenced by variations of the substratum, particularly ground water regime and soil chemical and textural properties (e.g. Newbery et al. 1986, ter
Steege et al. 1993, Duivervoorden 1996, Clark et al. 1998, Botrel et al. 2002). Therefore, the comparison between the two stand ages could not disregard possible interference from substratum-related variables. On a local scale, topographic variables (e.g. elevation, slope grade, plot aspect) have been regarded as the most important substratum-related variable causing spatial variation in the structure of tropical forests because it commonly corresponds to changes in soil properties, particularly drainage, texture and nutritional status (e.g. Bourgeron 1983, Newbery \& Proctor 1984, Clark et al. 1998). As this topographic pattern was present in every plot, we believe it caused no serious impact on the comparisons between the two stand ages. With respect to soil properties, organic matter was the only variable that differed between the two stands, with higher proportions in the younger one; the other variables showed similar variation across both stands. This outcome is rather unexpected because the common pattern in tropical secondary forests as regeneration proceeds is a progressive increase of the soil organic matter pool (e.g. Brown et al. 1984, Lugo et al. 1986), reaching levels similar to mature forests $40-50$ years after abandonment (Brown \& Lugo 1990). Possible reasons for this may involve both the massive initial litter input left by logging and the higher litterfall rates of the younger stand caused by a more rapid turnover and self-thinning process. However, data on litter input and

Table 5. Number of trees per species guilds in the two stands of secondary forest. Contingency tables and chi-square tests for the frequency of trees of each species guild in the 15- and 40-year old stands (expected values within brackets). $*=P<0.05$; $* *=P<0.001 ; \mathrm{ns}=$ non-significant.

\begin{tabular}{lccc}
\hline & \multicolumn{2}{c}{ Forest stand } & \\
\cline { 2 - 3 } Regeneration guilds & 15 -year old & 40 -year old & Chi-square tests \\
\hline Pioneer & $89(100.9)$ & $64(52.1)$ & $\chi^{2}=4.14 *$ \\
Light-demanding & $1105(986.9)$ & $391(509.1)$ & $\chi^{2}=41.55 \mathrm{~ns}$ \\
Shade-tolerant & $409(515.2)$ & $372(265.8)$ & $\chi^{2}=64.33 * *$ \\
Chi-square tests & $\chi^{2}=37.44 * *$ & $\chi^{2}=72.58 * *$ & $\chi^{2}=110.02 * *$ \\
Stratification guilds & & & \\
Large & $1031(996.1)$ & $479(513.9)$ & $\chi^{2}=3.59 \mathrm{~ns}$ \\
Medium & $526(524.4)$ & $269(270.6)$ & $\chi^{2}=0.01 \mathrm{~ns}$ \\
Small & $46(82.5)$ & $79(42.5)$ & $\chi^{2}=47.37 * *$ \\
Chi-square tests & $\chi^{2}=17.35 * *$ & $\chi^{2}=33.63 * *$ & $\chi^{2}=50.97 * *$ \\
Dispersion guilds & & $251(240.6)$ & $\chi^{2}=0.68 \mathrm{~ns}$ \\
Anemochorous & $456(466.4)$ & $97(99.0)$ & $\chi^{2}=0.06 \mathrm{~ns}$ \\
Autochorous & $194(192.0)$ & $479(487.4)$ & $\chi^{2}=0.22 \mathrm{~ns}$ \\
Zoochorous & $953(944.6)$ & $\chi^{2}=0.63, \mathrm{~ns}$ & $\chi^{2}=0.96 \mathrm{~ns}$ \\
Chi-square tests & $\chi^{2}=0.33, \mathrm{~ns}$ & & \\
\hline
\end{tabular}


decomposition rates, as well as on tree community dynamics, would be necessary to confirm this.

The total number of species registered in the two stands (199) is remarkably high compared to similar surveys of fragments of semideciduous forests of the Atlantic Forest Domain, which seldom surpass 140 species (database in Oliveira Filho \& Fontes 2000). The reason for these findings may lie in the origin of individual regenerating trees as a result of the local disturbancerecovery history (see Uhl \& Clark 1983, Murphy \& Lugo 1986). Logging operations were of low impact, using axes and cart-horses. The areas were abandoned shortly after the logs were removed and a considerable proportion of trees re-established, sprouting from stumps and roots. This was indicated by the high proportion of multi-stemmed trees, about $11 \%$, which contrasts with values below $4 \%$ found for mature forests in the region (authors' unpublished data). Also, there were always adjacent older stand sectors serving as seed sources. The combination of factors probably minimised the loss of species in the whole fragment.

The typical pattern of species diversity in tropical secondary forests is the accumulation of species, approaching that of a mature forest within a time span of up to 80 years (Brown \& Lugo 1990, Tabarelli \& Mantovani 1999). In accordance with this, the older stand yielded a higher number of species than did the younger stand. However, this was true only over larger areas (> 12 subplots) because the younger stand had higher species density (i.e. number of species per unit area) when smaller areas were compared. Some interactive factors may be involved in this inversion. Firstly, the higher tree density of the younger stand would contribute to increase species density at smaller areas. This relationship would not hold true, however, for larger areas because earlier regeneration phases would contain a smaller species pool than later phases. Secondly, the progression of forest regeneration could also enhance aspects of habitat differentiation related to the substratum and light environment, which are more relevant for species of later regeneration phases.

Despite an age difference of 25 years, the two stands yielded similar values of basal area and volume per hectare. This result is no surprise because tropical secondary forests usually undergo rapid accumulation of biomass during the first 15 years or so and then slow down, often reaching values of basal area and volume comparable to those of a mature forest many decades before maturity (Brown \& Lugo 1990). The live stumps and root systems left in the area after logging probably favoured a rapid accumulation of biomass, and the two stands may have reached similar values of basal area and volume before 15 years of age. They could be both continuing to accumulate biomass, though at lower rates. Tabarelli \& Mantovani (1999) recorded figures of basal area and volume in secondary Atlantic rain forests aged 40 years considerably below those of nearby mature forests, although the regeneration started from pastures without live tree remnants.

Tree density, particularly for smaller trees, was the most pronounced difference in vegetation structure between the two stand ages. This result agrees with the well-known self-thinning process of aging secondary forests in which a declining tree density, mostly caused by mortality rates concentrated on smaller trees $(\mathrm{dbh}<10 \mathrm{~cm})$, is highly compensated by the growth of surviving trees (e.g. Crow 1980, Saldarriaga et al. 1988, Rao et al. 1990, Brown \& Lugo 1990, Oliveira Filho et al. 1997). Therefore, as forest regeneration proceeds, the average tree size increases while tree density declines.

The structure of the tree species abundance data analysed by DCA contained a large amount of unexplained variance ('noise'). Nevertheless, a species gradient apparently related chiefly to the stands ages did emerge. This main gradient consisted mostly of variations in species abundances than species turnover, characterizing a short gradient, sensu ter Braak (1995). In fact, the two stand ages shared 44 out of the 47 species used in the analysis, and 98 out of the 199 species total (49\%). Despite this high floristic similarity, the two stands differed significantly on the relative abundance of many species. The interpretation of these patterns as a result of different regeneration ages deserves, however, some restriction because the differences may have resulted, at least in part, to contagious distribution patterns, which predominate among tropical forest tree species (Hubbell \& Foster 1986). Therefore, the differences in population abundances between the two stands could result from species clumps and, therefore, spatial autocorrelation. Despite this, there were other findings that fortunately weaken the power of this alternative hypothesis. The differences on the proportion of trees per regeneration and stratification guilds between the two stands provided additional substance to the differences in tree community composition.

Species which were more frequent than expected in the younger stand, such as Byrsonima sericea, Pera glabrata, Toulicia laevigata, Astronium fraxinifolium and Pimenta pseudocaryophyllus, are shadeintolerants commonly found in disturbed forests or at forest edges in the region. On the other hand, Bathysa 
nicholsonii, Eugenia neoglomerata, Siparuna guianensis, Astronium graveolens and Trichilia pallida, which were more frequent than expected in the older stand, are shade-tolerant species commonly found regenerating in the understorey of mature forests. As many shade-tolerant species are also typical of the forest understorey (e.g. Bathysa nicholsonii and Siparuna guianensis), the frequency of small trees was higher in the older stand. The significantly higher abundance of light-demanding and shade-tolerant species in the younger and older stand, respectively, also supports the view of two distinct regeneration phases. Species classification based on regeneration and stratification guilds have been successfully used in the analysis of tropical forest dynamics and regeneration in a number of studies, yielding similar patterns (e.g. Manokaran \& Kochumenn 1987, Whitmore 1989, Lieberman et al. 1990, Oliveira Filho et al. 1997, Tabarelli \& Mantovani 1999).

The two stands had similar proportions of trees per dispersion guild, failing to show an increasing abundance of zoochorous species with age found in Atlantic secondary forests by some authors (e.g. Klein 1980, Mendonça et al. 1992, Tabarelli \& Mantovani 1999). Two facts may explain this result. First, and quite likely, the predominance of anemochory could have occurred in earlier regeneration phases when wind-dispersed trees and shrubs (e.g. Toulicia laevigata, Astronium fraxinifolium, Eremanthus incanus and Aloysia virgata) established. Secondly, many abundant pioneer and light-demanding species are zoochorous (e.g. Byrsonima sericea, Pera glabrata, Guatteria villosissima, Cecropia hololeuca and Vismia guianensis) and may contribute substantially to the flora of early regeneration phases, as described by other authors (e.g. Gómez-Pompa et al. 1991, Vieira et al. 1996).

In general, tropical secondary forests restore species richness first and then diversity, species guild profile, species composition, and, finally, vegetation structure, particularly tree density and biomass, all within a time span between 50 and 150 years (e.g. Uhl et al. 1982, Saldarriaga et al. 1988, Tabarelli \& Mantovani 1999, Saldarriaga \& Uhl 1991). In the present case, species richness and diversity, as well as tree volume and basal area, were apparently the first characteristics of the two stands to converge. The relatively rapid restoration of these features was certainly fostered by tree regeneration from stumps and roots and by the existence of adjacent patches of old-growth forests operating as seed sources.
Acknowledgements - We wish to thank the Companhia Energética de Minas Gerais, Cemig, for full sponsorship of fieldwork and laboratory analyses. The first author thanks with gratitude the support received from the $\mathrm{CNPq}$ (research grant n. 301644/88-8). We are immensely grateful to our colleagues Valéria E.G. Rodrigues, and Paulo Emílio F. Mota, and to the students Warley A.C. Carvalho, Reginaldo S. Alves and Luciana Botezelli for their valuable help during field work, to Peter Hargreaves for carefully reviewing the English, and José Roberto Scolforo for his invaluable advise on the statistical analyses. We also thank two anonymous referees who contributed substantially to improve the manuscript.

\section{References}

APG II 2003. An update of the Angiospem Phylogeny Group classification for the orders and families of flowering plants: APG II. Botanical Journal of the Linnean Society 141:399-436.

BARROSO, G.M., MORIM, M.P., PEIXOTO, A.L. \& ICHASO, C.L.F. 1999. Frutos e sementes: morfologia aplicada à sistemática de dicotiledôneas. Editora UFV, Viçosa.

BOTREL, R.T., OLIVEIRA FILHO, A.T., RODRIGUES, L.A.\& CURI, N. 2002. Influência do solo e topografia sobre as variações da composição florística e estrutura da comunidade arbóreo-arbustiva de uma floresta estacional semidecidual em Ingaí, MG. Revista Brasileira de Botânica 25:195-213.

BOURGERON, P.S. 1983. Spatial aspects of vegetation structure. In Ecosystems of the world 14A - Tropical rain forest ecosystems, structure and function. (F.B. Golley, ed.). Elsevier, Amsterdam, p.29-47.

BROWN, S. \& LUGO, A.E. 1990. Tropical secondary forests. Journal of Tropical Ecology 6:1-32.

BROWN, S., GLUBCZYNSKI, A. \& LUGO, A.E. 1984. Effects of land use and climate on the organic carbon content of tropical forest soils in Puerto Rico. In New forests for a changing world (Society of American Foresters, ed.). Proceedings of the Society of American Foresters, Washington, D.C., p.204-209.

CARVALHO, D.A., OLIVEIRA FILHO, A.T., VILELA, E.A. \& CURI, N. 2000. Florística e estrutura da vegetação arbórea de um fragmento de floresta semidecidual às margens do reservatório da Usina Hidrelétrica Dona Rita (Itambé do Mato Dentro, MG). Acta Botanica Brasilica 14:37-55.

CLARK, D.B., CLARK, D.A. \& READ, J.M. 1998. Edaphic variation and the mesoscale distribution of tree species in a neotropical rain forest. Journal of Ecology 86:101-112.

CROW, T.R. 1980. A rainforest chronicle: a 30-year record of change in structure and composition at El Verde, Puerto Rico. Biotropica 12:42-55.

DUIVERVOORDEN, J.F. 1996. Patterns of tree species richness in rain forest of the Middle Caquetá area, Colombia, MW Amazonia. Biotropica 28:142-158. 
EMBRAPA. 1997. Manual de métodos de análises de solo. $2^{\text {nd }}$ ed. Empresa Brasileira de Pesquisa Agropecuária, Centro Nacional de Pesquisa de Solos, Rio de Janeiro.

GANDOLFI, S., LEITÃO FILHO, H.F. \& BEZERRA, C.L.F. 1995. Composição florística e estrutura fitossociológica de uma mata residual na área do Aeroporto Internacional de Cumbica (Guarulhos). Revista Brasileira de Biologia 55:753-767.

GÓMEZ-POMPA, A.G., WHITMORE, T.C. \& HADLEY, M. 1991. Tropical rain forests: regeneration and management. Blackwell, New York.

GUARIGUATA, M.R. \& DUPUY, J.M. 1997. Forest regeneration in abandoned logging roads in lowland Costa Rica. Biotropica 29:15-28.

HILL, M.O. \& GAUCH, H.G. 1980. Detrended correspondence analysis, an improved ordination technique. Vegetatio 42:47-58.

HUBBELL, S.P. \& FOSTER, R.B. 1986. Commonness and rarity in a Neotropical forest: implications for tropical tree conservation. In Conservation biology, the science of scarcity and diversity (M.E. Soulé, ed.). Sinauer, Sunderland, p.205-231.

KLEIN, R.M. 1980. Ecologia da flora e vegetação do Vale do Itajaí. Sellowia 32:165-389.

KORNING, J. \& BALSLEV, H. 1994. Growth and mortality of trees in Amazonian tropical rain forest in Ecuador. Journal of Vegetation Science 4:77-86.

KREBS, C.J. 1989. Ecological methodology. Harper and Row, New York.

LIEBERMAN, D., HARTSHORN, G.S., LIEBERMAN, M. \& PERALTA, R. 1990. Forest dynamics at La Selva Biological Station, 1969-1985. In Four Neotropical Rainforests (A.H. Gentry, ed.). Yale University Press, New Haven, p.509-521.

LORENZI, H. 1992. Árvores brasileiras: manual de identificação e cultivo de plantas arbóreas nativas do Brasil, v.1, Plantarum, Nova Odessa.

LORENZI, H. 1998. Árvores brasileiras: manual de identificação e cultivo de plantas arbóreas nativas do Brasil. v.2, Plantarum, Nova Odessa.

LUGO, A.E., SANCHEZ, M.J. \& BROWN, S. 1986, Land use and organic carbon content of sole subtropical soils. Plant and Soils 96:185-196.

MANOKARAN, N. \& KOCHUMMEN, K.M. 1987. Recruitment, growth and mortality of tree species in a lowland dipterocarp forest in Peninsular Malaysia. Journal of Tropical Ecology 3:315-330.

MENDONÇA, R.R., POMPÉIA, S.L. \& MARTINS, S.E. 1992. A sucessão secundária da Mata Atlântica na Região de Cubatão. In Anais do II Congresso Nacional sobre Essências Nativas, v. I (Instituto Florestal, ed.). Instituto Florestal, São Paulo, p.131-138.

MORELLATO, L.P.C. \& LEITÃO FILHO, H.F. 1992. Padrões de frutificação e dispersão na Serra do Japi. In História natural da Serra do Japi: ecologia e preservação de uma área no Sudeste do Brasil (L.P.C. Morellato, org.). Editora da Unicamp/Fapesp, Campinas, p.112-141.
MURPHY, P.G. \& LUGO, A.E. 1986. Ecology of tropical dry forest. Annual Review of Ecology and Systematics 17:67-88.

NEWBERY, D.M. \& PROCTOR, J. 1984. Ecological studies in four contrasting lowland rain forests in Gunugu Mulu National Park, Sarawak. IV. Association between tree distribution and soil factors. Journal of Ecology 72:475-493.

NEWBERY, D.M., GARTLAN, J.S., MCKEY, D.B. \& WATERMAN, P.G. 1986. The influence of drainage and soil phosphorus on the vegetation of Douala-Edea Forest reserve, Cameroun, Vegetatio 65:149-162.

NUNES, Y.R.F., MENDONÇA, A.V.R., BOTEZELLI, L., MACHADO, E.L.M. \& OLIVEIRA FILHO, A.T. 2003. Variação da fisionomia, diversidade e composição de guildas da comunidade arbórea em um fragmento de floresta semidecidual em Lavras, MG. Acta Botanica Brasilica 17:215-231.

OLIVEIRA FILHO, A.T. \& FONTES, M.A.L. 2000. Patterns of floristic differentiation among Atlantic forests in south-eastern Brazil, and the influence of climate. Biotropica 32:793-810.

OLIVEIRA FILHO, A.T., VILELA, E.A., CARVALHO, D.A. \& GAVILANES, M.L. 1994. Effects of soils and topography on the distribution of tree species in a tropical riverine forest in south-eastern Brazil. Journal of Tropical Ecology 10:483-508.

OLIVEIRA FILHO, A.T., MELLO, J.M. \& SCOLFORO, J.R.S. 1997. Effects of past disturbance and edges on tree community structure and dynamics within a fragment of tropical semideciduous forest in south-eastern Brazil over a five-year period (1987-1992). Plant Ecology 131:45-66.

OLIVEIRA FILHO, A.T., CURI, N., VILELA, E.A. \& CARVALHO, D.A. 2001. Variation in tree community composition and structure with changes in soil properties within a fragment of semideciduous forest in south-eastern Brazil. Edinburgh Journal of Botany 58:139-158.

PALMER, M.W. 1991. Estimating species richness: the second-order jackknife estimator reconsidered. Ecology 72:1512-1513.

PARTHASARATHY, N. 1999. Tree diversity and distribution in undisturbed and human-impacted sites of tropical wet evergreen forest in southern Western Ghats, India. Biodiversity and Conservation 8:1365-1381.

PESSOA, S.V.A., GUEDES-BRUNI, R.R. \& BRUNO, C.K. 1997. Composição florística e estrutura do componente arbustivo-arbóreo de um trecho secundário de floresta montana na Reserva Ecológica de Macaé de Cima. In Serra de Macaé de Cima: diversidade, florística e conservação em Mata Atlântica (H.C. Lima \& R.R. Guedes-Bruni, eds.). Jardim Botânico do Rio de Janeiro, Rio de Janeiro, p.147-168.

RAO, P., BARIK, S.K., PANDEY, H.N. \& TRIPATHI, R.S. 1990. Community composition and population structure in a sub-tropical broad-leaved forest along a disturbance gradient. Vegetatio 88:151-162. 
SALDARRIAGA, J.G. \& UHL, C. 1991. Recovery of forest vegetation following slash-and-burn agriculture in the upper Rio Negro. In Tropical rain forests: regeneration and management (A. Gómez-Pompa, T.C. Whitmore \& M. Hadley, eds.). Blackwell, New York, p.303-312.

SALDARRIAGA, J.G., WEST, D.C. \& THARP, M.L. 1988. Long-term chronosequence in the upper Rio Negro of Colombia and Venezuela. Journal of Ecology 76:938-958.

SOS MATA ATLÂNTICA. 1998. Atlas da evolução dos remanescentes florestais e ecossistemas associados no domínio da Mata Atlântica no Período 1990-1995. Fundação SOS Mata Atlântica, São Paulo.

SOS MATA ATLÂNTICA \& INPE. 1993. Evolução dos remanescentes florestais e ecossistemas associados do domínio da Mata Atlântica. Fundação SOS Mata Atlântica and Instituto Nacional de Pesquisas Espaciais, São Paulo.

SWAINE, M.D. \& WHITMORE, T.C. 1988. On the definition of ecological species groups in tropical rain forests. Vegetatio 75:81-86.

TABARELLI, M. \& MANTOVANI, W. 1999. A regeneração de uma floresta tropical montana após corte e queima (São Paulo, Brasil). Revista Brasileira de Biologia 59:239-250.

TER BRAAK, C.J.F. 1995. Ordination. In Data analysis in community and landscape ecology (R.H.G. Jongman, C.J.F. ter Braak \& O.F.R. van Tongeren, eds.). Cambridge University Press, Cambridge, p.91-173.

TER BRAAK, C.J.F. \& ŠMILAUER, P. 1998. CANOCO Reference manual and user's guide to CANOCO for Windows: software for canonical community ordination (version 4). Microcomputer Power, Ithaca.
TER STEEGE, H., JETTEN, V.G., POLAK, A.M. \& WERGER, M.J.A. 1993. Tropical rain forest types and soil factors in a watershed area in Guyana. Journal of Vegetation Science 4:705-716.

UHL, C. \& CLARK, K. 1983. Seed ecology of selected Amazon Basin successional species emphasizing forest seed banks, seed longevity, and seed germination triggers. Botanical Gazette 144:419-425.

UHL, C., JORDAN, C., CLARK, K., CLARK, H. \& HERRERA, R. 1982. Ecosystem recovery in Amazon caatinga forest after cutting, cutting and burning, and bulldozer clearing treatments. Oikos 38:313-320.

VAN DER PIJL, L. 1982. Principles of dispersal in higher plants. $3^{\text {rd }}$ ed. Springer-Verlag, Berlin.

VAN TONGEREN, O.F.R. 1995. Cluster analysis. In Data analysis in community and landscape ecology (R.H.G. Jongman, C.J.F. ter Braak \& O.F.R. van Tongeren, eds.). Cambridge University Press, Cambridge, p.174-212.

VIANA, V.M. \& TABANEZ, A.A.J. 1996. Biology and conservation of forest fragments in the Brazilian Atlantic moist forest. In Forest patches in tropical landscapes (J. Schelhas \& R. Greengberg, eds.). Island Press, Washington, D.C., p.151-167.

VIEIRA, I.C.G., SALOMÃO, R.P., ROSA, N.A., NEPSTADT, D.C. \& ROMA, J.C. 1996. O renascimento da floresta no rastro da agricultura. Ciência Hoje 20:38-44.

WHITMORE, T.C. 1989. Changes over twenty-one years in the Kolombangara rain forests. Journal of Ecology 77:469-483.

WHITMORE, T.C. 1990. An introduction to tropical rain forests. Blackwell, London.

ZAR, J.H. 1996. Biostatistical analysis. Prentice-Hall, New Jersey. 